\title{
XMM-Newton observation of an unusual iron line in the quasar Markarian 205
}

\author{
J. N. Reeves ${ }^{1}$, M. J. L. Turner ${ }^{1}$, K. A. Pounds ${ }^{1}$, P. T. O’Brien ${ }^{1}$, Th. Boller ${ }^{2}$, P. Ferrando ${ }^{3}$, E. Kendziorra ${ }^{4}$, \\ and S. Vercellone ${ }^{5}$ \\ 1 X-Ray Astronomy Group, Department of Physics and Astronomy, Leicester University, Leicester LE1 7RH, UK \\ 2 Max-Planck-Institut für extraterrestrische Physik, Postfach 1603, 85748 Garching, Germany \\ 3 Service d'Astrophysique, CEA Saclay, 91191 Gif-sur-Yvette, France \\ ${ }^{4}$ Institut für Astronomie und Astrophysik - Astronomie, University of Tübingen, Waldhäuser Strasse 64, \\ 72076 Tübingen, Germany \\ 5 Istituto di Fisica Cosmica "G. Occhialini", C.N.R., Via Bassini 15, 20133 Milano, Italy
}

Received 28 September 2000 / Accepted 20 October 2000

\begin{abstract}
XMM-Newton observations of the low luminosity, radio-quiet quasar Markarian 205 have revealed a unique iron $\mathrm{K}$ emission line profile. In marked contrast to the broad and redshifted iron $\mathrm{K}$ line commonly seen in $A S C A$ observations of Seyfert 1 galaxies, we find that a substantial amount of the line flux in Mrk 205 occurs above the neutral line energy of $6.4 \mathrm{keV}$. Furthermore, we find that the iron line emission has two distinct components, a narrow, unresolved neutral line at $6.4 \mathrm{keV}$ and a broadened line centred at $6.7 \mathrm{keV}$. We suggest that the most likely origin of the $6.7 \mathrm{keV}$ line is from X-ray reflection off the surface of a highly ionised accretion disk, whilst the $6.4 \mathrm{keV}$ component may arise from neutral matter distant from the black hole, quite possibly in the putative molecular torus. Overall this observation underlines the potential of XMM-Newton for using the iron $\mathrm{K}$ line as a diagnostic of matter in the innermost regions of AGN.
\end{abstract}

Key words. galaxies: active - quasars: individual: Markarian 205 - X-rays: galaxies

\section{Introduction}

Since iron $\mathrm{K} \alpha$ emission was found to be a common feature in the X-ray spectra of AGN, it has been recognised as potentially a very important probe of matter in the central nucleus. Observations with the Ginga X-ray satellite first established the iron $\mathrm{K} \alpha$ line (at $6.4 \mathrm{keV}$ ) as a dominant feature in the hard X-ray spectra of many broad-line Seyfert 1 galaxies (Pounds et al. 1990; Nandra \& Pounds 1994). The line was often accompanied by a flattening of the X-ray continuum (the Compton scattering hump) above the line energy. A widely favoured interpretation of these spectral features, as primary hard X-rays being "reflected" by Compton-thick matter - evidence for the putative accretion disk in AGN - was strengthened when $A S C A$, with its higher resolution solid-state detectors, was able to resolve the iron $\mathrm{K}$ emission line. The line profile of the bright Seyfert 1 galaxy MCG -6-30-15 (Tanaka et al. 1995) was found to be broadened $(v \sim 0.3 c)$, with a strong asymmetry red-wards of $6.4 \mathrm{keV}$; the extreme width and redshift of the iron line being attributed to the strong gravity and high velocities of the matter in the innermost regions around the putative massive black hole. Subsequent observations of a sample of Seyfert 1s with
$A S C A$ (Nandra et al. 1997) revealed that such line profiles were apparently common in many nearby Seyfert 1s.

In contrast, the information on the more luminous, but generally fainter, quasars has remained inconclusive. Ginga detected iron K emission from only a few quasars (Williams et al. 1992; Lawson et al. 1997), whilst although $A S C A$ increased the number with detected iron lines (Reeves et al. 1997; Reeves \& Turner 2000), the data were not sufficient to resolve any of the line profiles. Despite this, a divergence in iron K line properties between the low luminosity Seyfert 1 galaxies and higher luminosity quasars has become apparent, with the line energy being closer to $6.7 \mathrm{keV}$, rather than $6.4 \mathrm{keV}$, and the line strength falling with increasing luminosity (Iwasawa \& Taniguichi 1993; Nandra et al. 1997b). The sensitivity of the XMM-Newton EPIC instruments now offers the exciting prospect of observing the iron $\mathrm{K}$ emission of quasars in detail and hence studying the flow and physical state of matter in the nuclei of these most powerful of objects.

This paper presents the discovery, by XMM-Newton, of an unusual iron $\mathrm{K} \alpha$ line profile in the quasar Markarian 205, observed during the Cal-PV (Calibration and Payload Verification) phase. Mrk 205 is a nearby $(z=0.071)$, low luminosity quasar $\left(M_{V}=-23\right.$, 
$L_{\mathrm{X}}=210^{44} \mathrm{erg} / \mathrm{s}, L_{\mathrm{BOL}} \sim 510^{45} \mathrm{erg} / \mathrm{s} ;$ Williams et al. 1992; Reeves \& Turner 2000) and is radio-quiet (Rush et al. 1996). It is located only $0.7^{\prime}$ away from the nearby $(z=0.00468)$ spiral galaxy NGC 4319 and is viewed through the outer disk of this galaxy (Bahcall et al. 1992; Bowen \& Blades 1993). Mrk 205 is also unusual in that it has a very weak UV bump (McDowell et al. 1989), suggesting that the thermal disc emission is hidden in the EUV. We now present the XMM-Newton observations of Mrk 205. Values of $H_{0}=50 \mathrm{~km} \mathrm{~s}^{-1} \mathrm{Mpc}^{-1}$ and $q_{0}=0.5$ are assumed and all fit parameters are given in the quasar rest-frame.

\section{XMM-Newton observations}

Mrk 205 was observed during orbit 75 of the Cal-PV phase of XMM-Newton. The observations with the EPIC MOS (Turner et al. 2001) and PN (Strüder et al. 2001) detectors were split into 3 parts, each of $\sim 17 \mathrm{ksec}$ duration, to test a variety of sub-window modes. Three observations each were made with the MOS 1 and 2 cameras, in Full Window, Partial Window 2 and Partial Window 3 modes. For the PN, two observations were made in Full Window mode and one in Large Window mode. The data were screened with the XMM SAS (Science Analysis Software) and pre-processed using the latest CCD gain values known at the time of the observation. X-ray events corresponding to patterns $0-12$ for the 2 MOS cameras (similar to grades 0-4 in $A S C A$ ) were used; for the $\mathrm{PN}$, only pattern 0 events (single pixel events) were selected. Additional electronic noise was also removed from the MOS detectors. A low energy cut of $200 \mathrm{eV}$ was applied and known hot or bad pixels were removed during screening. The non X-ray background remained low throughout the observations. A screened MOS-1 image of Mrk 205 is shown in Fig. 1.

We then proceeded to extract spectra and light curves for both the source and background in each of the three observations, separately for each EPIC detector. A circular source region of $1^{\prime}$ diameter was defined around the centroid position of Mrk 205, with the background being taken from an offset position close to the source. This resulted in 3 source spectra, for each of the 3 EPIC CCD cameras. There was little flux or spectral variability of Mrk $205(<10 \%)$ during the observations. Indeed a spectral fit of a power-law plus Gaussian emission line (near to $6.4 \mathrm{keV}$ ) yielded consistent results for each of the three observations. Therefore we proceeded to co-add the 3 observations, for each detector. Furthermore as there was little difference between the MOS 1 and MOS 2 spectra (both in the spectral shape and the iron line profile described later), we combined these into a single spectral file to maximise signal-to-noise. The resultant process left one combined EPIC-MOS spectrum, with a total exposure of $100 \mathrm{ksec}$ (both MOS, co-added) and one PN spectrum, with a total exposure of $44 \mathrm{ksec}$. The integrated X-ray flux from Mrk 205, over the $0.5-10 \mathrm{keV}$ range, was $810^{-12} \mathrm{ergs} \mathrm{cm}^{-2} \mathrm{~s}^{-1}$. At this flux level, the effect of photon pile-up, even in MOS full-frame mode, is

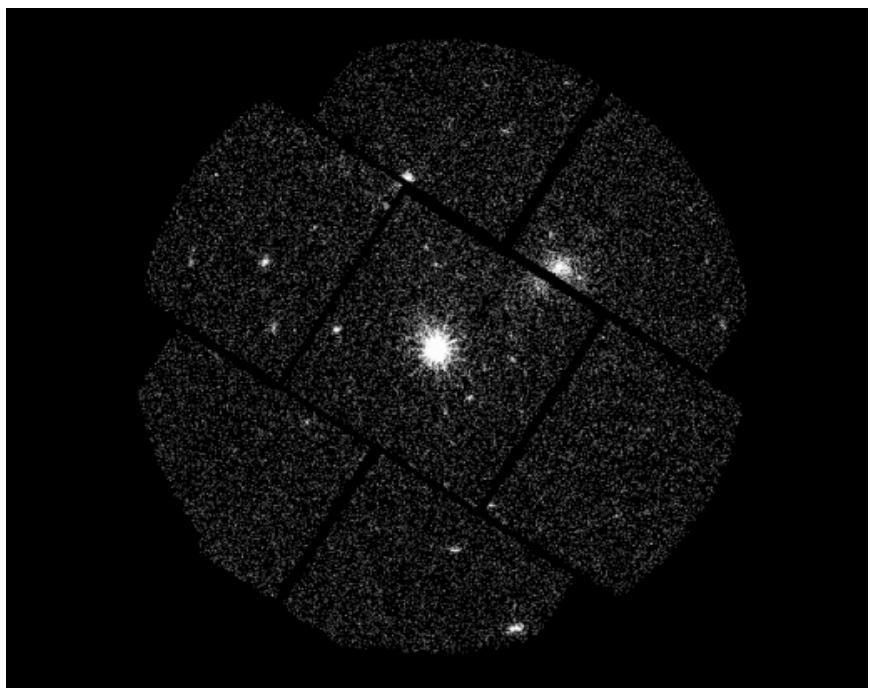

Fig. 1. An EPIC MOS-1 image of the Mrk 205 observation. Mrk 205 is located in the centre, the extended source near the chip gap is the nearby elliptical galaxy NGC 4291

negligible. The background subtracted spectra were fitted, using XSPEC V11.0, with the latest response matrices produced by the EPIC team; the systematic level of uncertainty is $<5 \%$. Finally spectra were binned to a minimum of 20 counts per bin, to apply the $\chi^{2}$ minimalisation technique.

\section{Spectral analysis - the iron $\mathrm{K}$ line}

We initially fitted the hard X-ray $(2.5-10 \mathrm{keV})$ spectrum of Mrk 205 with a power law and neutral absorption corresponding to the line-of-sight column through our Galaxy, of $N_{\mathrm{H}}=2.810^{20} \mathrm{~cm}^{-2}$ (Elvis et al. 1989). From our initial inspection of the EPIC spectra, it was obvious that deviations were present between $6-7 \mathrm{keV}$ suggestive of iron $\mathrm{K}$ shell band emission. We then proceeded to parameterise the profile of the observed feature in terms of simple Gaussian models for both the MOS and PN (see Table 1 for a summary of fits). Note all errors are quoted at the $90 \%$ confidence level.

Initially we fitted the MOS and PN data separately, to check for consistency between the 2 datasets. For the MOS, the addition of a narrow $(\sigma=0.01 \mathrm{keV})$ iron $\mathrm{K}$ line at $\sim 6.4 \mathrm{keV}$, to the underlying power-law, improved the fit significantly $\left(\Delta \chi^{2}=32.4\right.$ for 2 parameters). Even after addition of a narrow iron line, there were still residuals present at energies $>6.4 \mathrm{keV}$. Therefore we added a second line component, with the energy and line width both free to vary. The fit improved still further $\left(\Delta \chi^{2}=10.1\right.$ for 3 extra parameters $)$, with the overall fit-statistic of $\chi_{\nu}^{2}=363.5 / 341$ (fit 1 in Table 1). The narrow line energy is consistent with neutral iron $(E=6.39 \pm 0.04 \mathrm{keV})$, whilst the "broad" Fe line has 
Table 1. Fits to iron $\mathrm{K}$ line profile, in $2.5-10 \mathrm{keV}$ EPIC band. ${ }^{a}$ Energy of line in keV. ${ }^{b}$ Equivalent width of line in eV. ${ }^{c}$ Intrinsic velocity width of line (in $\mathrm{eV}$ ) or disk inclination angle. ${ }^{d}$ Improvement in $\chi^{2}$ for adding line component. ${ }^{f}$ Indicates parameter value is fixed in the fit. Note $\mathrm{PL}=$ Power-law; GA $=$ Gaussian; DISK $=$ Disk-line. For the disk-line, $R_{\text {in }}=6 R_{\mathrm{g}}$, $R_{\text {out }}=1000 R_{\mathrm{g}}$ and emissivity $\propto r^{-2.5}$ are all assumed

\begin{tabular}{|c|c|c|c|c|c|c|c|c|c|}
\hline \multirow[t]{2}{*}{$\overline{\text { Fit }}$} & \multirow[t]{2}{*}{ Instrument } & \multicolumn{3}{|c|}{ Narrow-Line } & \multicolumn{4}{|c|}{ Broad/Disk Line } & \multirow[t]{2}{*}{$\chi^{2} /$ dof } \\
\hline & & $E^{a}$ & $E W^{b}$ & $\Delta \chi^{2 d}$ & $E^{a}$ & $\sigma$ or $\theta^{c}$ & $E W^{b}$ & $\Delta \chi^{2 d}$ & \\
\hline 1. $\mathrm{PL}+2 \times \mathrm{GA}$ & MOS only & $6.39 \pm 0.04$ & $75 \pm 35$ & 32.4 & $6.68 \pm 0.22$ & $300_{-180}^{+280}$ & $125_{-75}^{+95}$ & 10.1 & $\overline{363.5 / 341}$ \\
\hline 2. $\mathrm{PL}+2 \times \mathrm{GA}$ & PN only & $6.46_{-0.08}^{+0.02}$ & $68 \pm 23$ & 23.7 & $6.78_{-0.16}^{+0.12}$ & $200_{-120}^{+300}$ & $140_{-55}^{+110}$ & 21.7 & $786 / 789$ \\
\hline 3. $\mathrm{PL}+2 \times \mathrm{GA}$ & $\mathrm{PN}+\mathrm{MOS}$ & $6.39 \pm 0.03$ & $56 \pm 23$ & 53 & $6.74 \pm 0.12$ & $250_{-130}^{+190}$ & $135_{-60}^{+70}$ & 30 & $1095 / 1093$ \\
\hline 4. PL + DISK & $\mathrm{PN}+\mathrm{MOS}$ & - & - & - & $6.4^{f}$ & $45^{\circ f}$ & $215_{-50}^{+80}$ & 56 & $1122 / 1096$ \\
\hline 5. PL + DISK & $\mathrm{PN}+\mathrm{MOS}$ & - & - & - & $6.60 \pm 0.06$ & $42 \pm 8^{\circ}$ & $300_{-70}^{+65}$ & 68 & $1110 / 1095$ \\
\hline 6. $\mathrm{PL}+\mathrm{GA}+\mathrm{DISK}$ & $\mathrm{PN}+\mathrm{MOS}$ & $6.39 \pm 0.04$ & $46 \pm 19$ & 16 & $6.67 \pm 0.10$ & $37 \pm 12^{\circ}$ & $225_{-105}^{+75}$ & 68 & $1094 / 1093$ \\
\hline \multirow[t]{2}{*}{ 7. $\mathrm{PL}+3 \times \mathrm{GA}$} & $\mathrm{PN}+\mathrm{MOS}$ & $6.39 \pm 0.03$ & $70 \pm 18$ & 50 & $6.59 \pm 0.06$ & $10^{f}$ & $35 \pm 17$ & 14 & \\
\hline & & & & & $6.85 \pm 0.05$ & $10^{f}$ & $51 \pm 19$ & 19 & $1095 / 1092$ \\
\hline
\end{tabular}

a higher energy of $6.7 \pm 0.2 \mathrm{keV}$ and an rms width of $\sigma=300 \mathrm{eV}$ (Fig. 2).

Next we fitted the PN data, which provided still tighter constraints on the $\mathrm{Fe} \mathrm{K}$ emission. A significant improvement in the simple power law fit $\left(\Delta \chi^{2}=23.7\right.$ for 2 parameters) was again obtained on adding a narrow line near $6.4 \mathrm{keV}$. As for the MOS, the fit was further improved $\left(\Delta \chi^{2}=21.7\right.$ for 3 extra parameters) by adding a second broad Fe line component (fit 2 in Table 1). Again the energy of the narrow line is consistent with neutral iron $\left(E=6.46_{-0.08}^{+0.02} \mathrm{keV}\right)$, whereas the energy of the "broad" line is near to $6.7 \mathrm{keV}\left(E=6.78_{-0.16}^{+0.12} \mathrm{keV}\right)$. The PN line profile, for the double Gaussian model, is also plotted in Fig. 2.

As the line spectral fits for the MOS and PN are fully consistent, we proceeded to fit the 2 spectra simultaneously, allowing for a slight $(<5 \%)$ normalisation difference between the 2 detectors. The addition of both the narrow $6.4 \mathrm{keV}$ line and the broader line at $6.7 \mathrm{keV}$ were both highly significant (for the narrow line, $\Delta \chi^{2}=53$ for 2 parameters; broad line, $\Delta \chi^{2}=30$ for 3 parameters) with the overall fit-statistic being very good $\left(\chi_{\nu}^{2}=1095.5 / 1093\right.$, fit 3 ). The narrow line is consistent with emission from neutral iron $(E=6.39 \pm 0.02 \mathrm{keV}$, equivalent width $E W=56 \pm 23 \mathrm{eV}$ ), whilst the broader line corresponds to ionised matter $\left(E=6.74 \pm 0.12 \mathrm{keV}, \sigma=250_{-130}^{+190} \mathrm{eV}\right.$ and $\left.E W=135_{-60}^{+65} \mathrm{eV}\right)$. We do not detect an iron $\mathrm{K}$ edge, the upper-limit on the edge, constrained between 7 and $9 \mathrm{keV}$, is $\tau<0.2$. However, we cannot rule out the presence of a continuum reflection component, from either neutral or moderately ionised material, with a covering fraction of 0.5 (corresponding to $R=\Omega / 2 \pi=1$ ). Note the best-fit power-law index of $\Gamma=1.80 \pm 0.04$ is consistent with other radio-quiet quasars (Reeves et al. 1997). Finally we plot confidence contours between line energy and normalisation for both lines in Fig. 3, illustrating the separate detection (at $>99.9 \%$ confidence) of both the narrow line at $6.4 \mathrm{keV}$ and the broad, blue-shifted component at $6.7 \mathrm{keV}$.
EPIC-MOS Fe line profile of Mrk 205

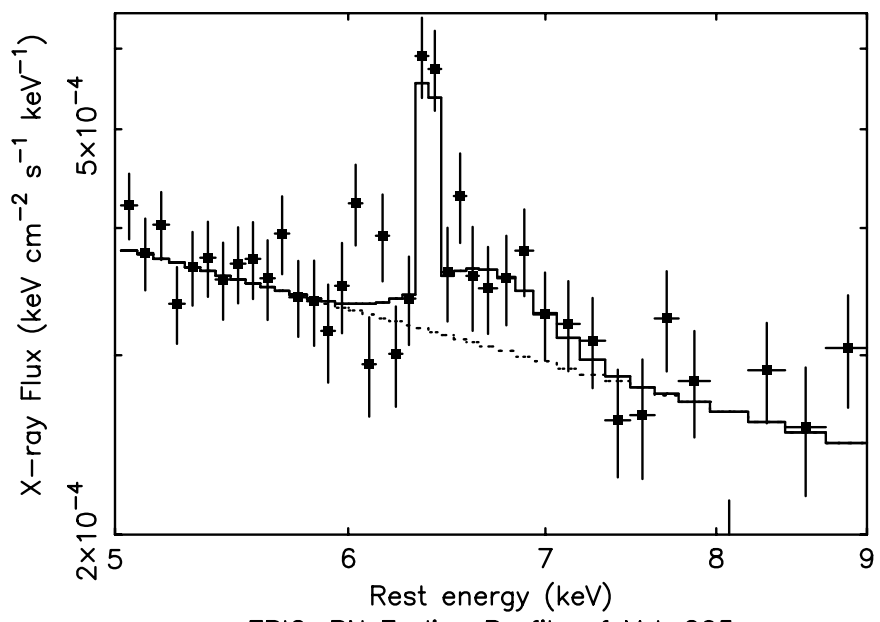

EPIC-PN Fe line Profile of Mrk 205

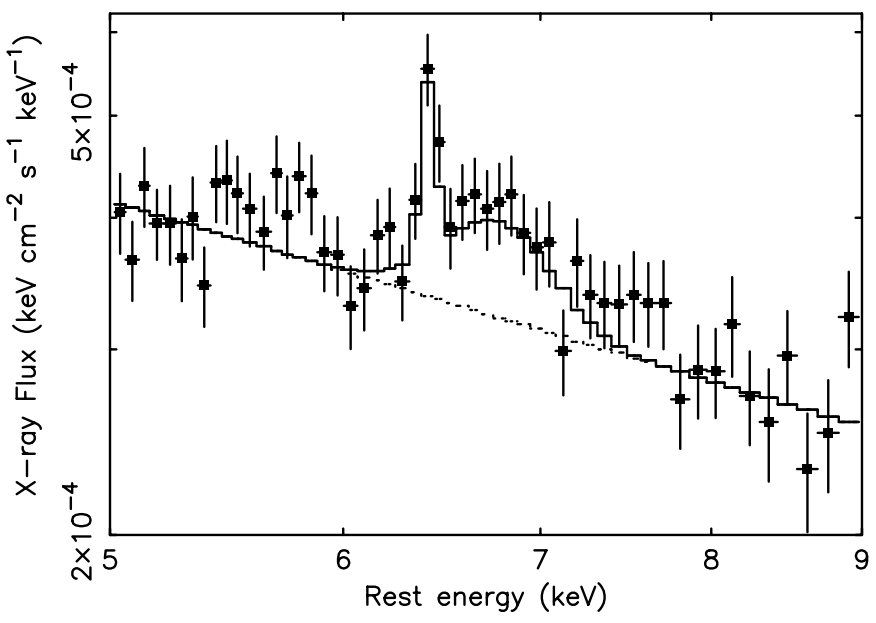

Fig. 2. The EPIC Fe line profile of Mrk 205, from the MOS and PN. A broad line component at $6.7 \mathrm{keV}$ is present, as well as a narrow emission line at $6.4 \mathrm{keV}$. The line profile differs considerably from those observed in Seyfert 1 galaxies (MCG -6-30-15, Tanaka et al. 1995; NGC 3516, Nandra et al. 1999), where most of the line flux is redshifted below $6.4 \mathrm{keV}$ 


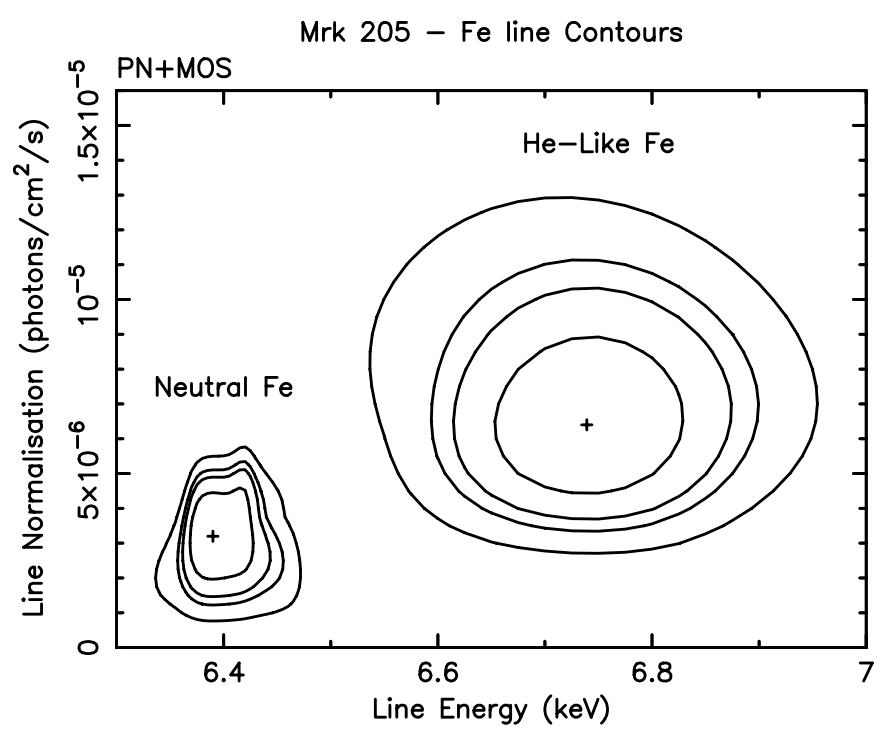

Fig. 3. Confidence contour plot for the double Gaussian Fe line profile. Contours correspond to the $68 \%, 90 \%, 99 \%$ and $99.9 \%$ confidence levels respectively. Clearly both the narrow line at $6.4 \mathrm{keV}$ and the broad component at $6.7 \mathrm{keV}$ are detected at a high level of significance

\subsection{Complex fits to the iron $K$ line profile}

Given the complexity of the Fe line in Mrk 205, we checked whether this could be reconciled with a relativistic diskline profile (Fabian et al. 1989). In the first trial, the rest-frame energy of the disk-line was fixed at $6.4 \mathrm{keV}$, appropriate for neutral iron (i.e. $<$ Fe Xx). This yielded a poor fit to the observed profile (fit 4, Table 1), for a disk inclination of $\sim 45^{\circ}$. This is not surprising as the diskline model requires that most of the line flux is located below $6.4 \mathrm{keV}$, which is clearly not observed. Next we freed the rest-frame energy in the disk-line model (fit 5). This yielded a better fit $\left(\Delta \chi^{2}=12\right)$, with an increased line energy of $E=6.60 \pm 0.06 \mathrm{keV}$, consistent with a higher ionisation of iron. However inspection of the line residuals indicated significant emission still remaining at $6.4 \mathrm{keV}$. Adding a second narrow, neutral iron line to the model (fit 6 ) did produce an acceptable fit, with the diskline rest energy at $6.67 \pm 0.10 \mathrm{keV}$. Thus the observations are only consistent with a diskline origin if the disk ionisation is high enough to produce He-like iron at $\sim 6.7 \mathrm{keV}$ and if the narrow, neutral Fe line component is also present.

Note that if the $6.7 \mathrm{keV}$ line component does originate from an ionised accretion disk, then one might still expect to see some line flux redshifted below $6.4 \mathrm{keV}$. We therefore tried to add a third, broad emission line component, with the energy constrained between 5 and $6 \mathrm{keV}$. However we were only able to place an upper-limit on this redshifted component $(E W<45 \mathrm{eV})$, and although the flux is substantially lower it is consistent with the amount of flux expected from our diskline fit to the ionised line. Finally we checked whether a blend of several narrow lines, from various ionisation species of iron, can model the data (fit 7 ). This did produce an acceptable fit $\chi_{\nu}^{2} \sim 1$ for three

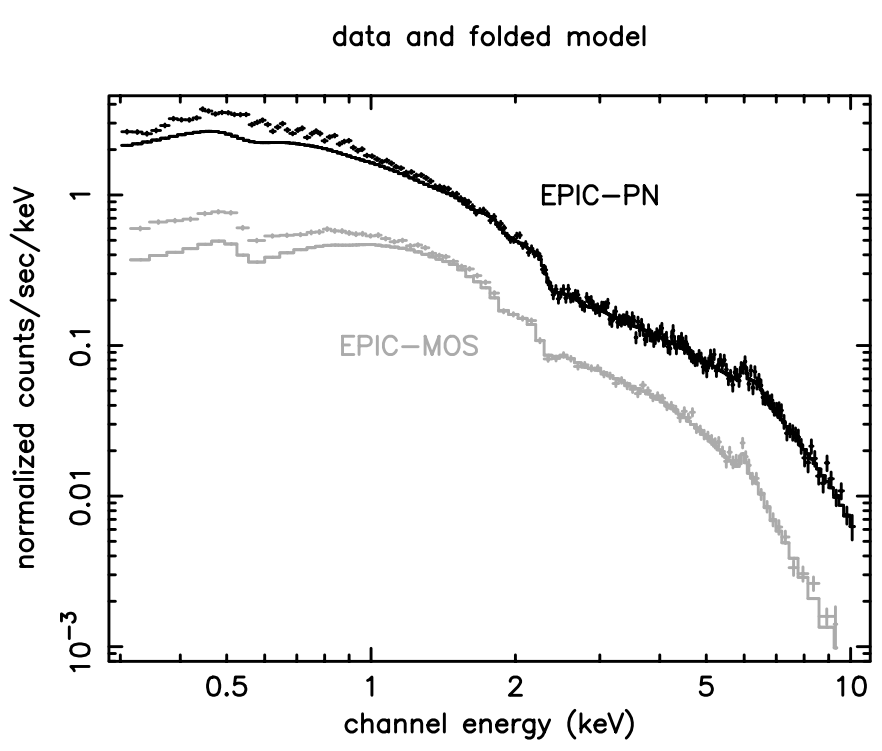

Fig. 4. The EPIC spectrum of Mrk 205, extrapolated to lower energies $(0.3 \mathrm{keV})$. An excess of soft X-ray counts, above that of the hard power-law, is clearly present in the data

narrow lines, with the best-fit line energies at $6.4 \mathrm{keV}$, $6.6 \mathrm{keV}$ and $6.85 \mathrm{keV}$, compatible with a line blend from neutral, He-like and H-like iron, respectively. We now defer further discussion of the iron emission until Sect. 5 .

\section{The broad-band XMM spectrum}

We now consider the broad-band EPIC spectrum of Mrk 205. Extrapolating the best-fit hard X-ray spectrum, with a power-law index of $\Gamma=1.8$, to lower energies gives a poor fit; there is a clear excess of soft X-ray counts above that of the hard X-ray power-law slope (Fig. 4). Refitting the continuum with a single power-law (with Galactic absorption) still gives a poor fit $\left(\chi_{\nu}^{2}=1.5,1630\right.$ channels $)$, albeit with a steeper photon index $(\Gamma=1.96 \pm 0.01)$. An acceptable reduced chi-squared $\left(\chi_{\nu}^{2}=1.2\right)$ is obtained on adding a black-body component to model the soft excess, with temperature $k T=120 \pm 8 \mathrm{eV}$ and $\Gamma=1.86 \pm 0.02$. The soft excess is relatively weak, representing only $\sim 15 \%$ of the emission over the range $0.5-2 \mathrm{keV}$.

Given the apparent weakness of the soft X-ray excess in Mrk 205, and its relatively high temperature $\left(T=1.510^{6} \mathrm{~K}\right)$, it is unlikely that we are observing the direct thermal emission from the disk in this bandpass (Malkan \& Sargent 1982). A more likely origin of the soft excess in Mrk 205 is by enhanced reflection of the primary power law continuum in the surface layers of an ionised accretion disk. Fitting the broad-band X-ray continuum of Mrk 205 with a hard power-law plus the reflected contribution from an ionised disk (using the PEXRIV model in XSPEC; Magdziarz \& Zdziarski 1995) provides a very good fit, including all of the soft X-ray excess. The parameters of the fit are also reasonable; $R=\Omega / 2 \pi=$ $1.1 \pm 0.2$, ionisation parameter $\xi=300 \pm 80 \mathrm{erg} \mathrm{cm} \mathrm{s}{ }^{-1}$ and $\Gamma=2.0 \pm 0.02$. Furthermore this emission could naturally explain the ionised part (at $6.7 \mathrm{keV}$ ) of the iron line 
profile; He-like Fe $\mathrm{K}$ emission from the disc is predicted in this range of ionisation (Ross et al. 1999; Nayakshin et al. 2000). Note that the detection of both the narrow and broad Fe line components is still robust (at $>99.9 \%$ confidence), even after the addition of reflection to the continuum.

Aside from the Fe $\mathrm{K}$ line emission, the EPIC X-ray spectrum of Mrk 205 appears to be devoid of other discrete emission or absorption features. To check this we constructed a high resolution RGS spectrum (from RGS1 and RGS2), using the RGSPROC script in the SAS software. The RGS spectra are in agreement with the EPIC data, the soft X-ray $(0.4-2 \mathrm{keV})$ spectrum of Mrk 205 is essentially featureless. We place limits on the strength of the OVII and OVIII edges, expected from a warm absorber, of $\tau<0.12$ and $\tau<0.15$ respectively. Furthermore we find no evidence for neutral photoelectric absorption, other than Galactic; the limit on $N_{\mathrm{H}}$ is $<1.510^{20} \mathrm{~cm}^{-2}$. Thus we are apparently observing the bare quasar nucleus in Mrk 205. There is also no measurable absorption from the intervening galaxy NGC 4319, consistent with the observation of a very highly ionised ISM in the spiral arms of that galaxy (Bowen \& Blades 1993).

\section{The origins of the line in Mrk 205}

We have found two separate components in the Fe K line profile of the quasar Mrk 205, observed with XMMNewton. A narrow line at $6.4 \mathrm{keV}$ is seen, presumably arising from neutral material in the quasar rest-frame, as well as a broad component at $6.7 \mathrm{keV}$. This observation provides the clearest example to date, from CCD-resolution data, of a distinct narrow Fe-K line component in a broadlined, type I AGN. We now consider the likely physical origins of the iron line components.

The narrow line at $6.4 \mathrm{keV}$. Detection of a strong, narrow iron emission line at $6.4 \mathrm{keV}$ implies that a substantial quantity of cool reprocessing material is present. This matter has to lie outside of the line-of-sight - the $\mathrm{X}$-ray spectrum is completely unabsorbed - and therefore is presumably seen by reflection. The line strength $(E W \sim 50-75 \mathrm{eV})$, implies that the cool matter subtends a substantial solid angle, of at least $1 \pi$ steradian, assuming it is Thomson thick and has a solar abundance of iron (e.g. George \& Fabian 1991). The most likely location of such material, within the framework of current AGN models, would appear to be the molecular torus (Antonucci 1993), with hard X-rays from the central engine being reflected off the inner surface of the torus and into the line-of-sight of the observer (Krolik et al. 1994). Strong iron $\mathrm{K} \alpha$ lines are believed to be formed in this way in Seyfert 2 galaxies (Turner et al. 1997), where the $\mathrm{X}$-ray continuum is at least partly obscured by the putative torus. Clear evidence for this torus emission has hitherto not been found in a quasar, where the (un-obscured) continuum level is much higher. Our observation of a narrow $6.4 \mathrm{keV}$ line from Mrk 205 could provide the first such evidence.
Although reflection off the putative torus is attractive, we cannot rule out other alternatives. Emission from BLR clouds could account for some of the narrow line flux, although the predicted line strength is small $(E W<50 \mathrm{eV}$; Leahy \& Creighton 1993). Interestingly, a high resolution Chandra-HETG observation has also resolved a "narrow" Fe K line in the Seyfert 1 NGC 5548 (Yaqoob et al. 2000), here the authors attribute the line to BLR gas, but they cannot exclude the possibility of a torus component. Another possibility is that the line originates by X-ray reflection from the outer regions of the accretion disc. Normally the angle subtended by the outer disk, to the X-ray source, would be small. However a warped, concave disk could increase the amount of reflection at large radii, and account for a measurable narrow line component at $6.4 \mathrm{keV}$ (Blackman 1999).

The broad line at $6.7 \mathrm{keV}$. The most obvious explanation for the broad line in Mrk 205 is that it originates from the inner accretion disc, where the material velocities are high, as well as a strong gravity gradient. However, the energy of the line emission is not consistent with the diskline profiles observed in many Seyfert 1s (Nandra et al. 1997), where the line flux is redshifted below $6.4 \mathrm{keV}$. Invoking a large inclination angle $\left(\theta=75-90^{\circ}\right)$ can circumvent this, but requires that the disc is orientated near edge-on, which appears to violate current AGN unification ideas (Antonucii 1993). An alternative is that the disc matter is in a high state of ionisation (with $\mathrm{He}$ or even $\mathrm{H}$-like $\mathrm{Fe}$ ). This is a plausible explanation and we note high ionisation Fe lines near $6.7 \mathrm{keV}$ have been claimed from $A S C A$ data in several radio-quiet quasars (Reeves \& Turner 2000), perhaps indicating a level of inner disc ionisation increasing with accretion rate (Matt et al. 1999). Indeed the red-wing of the Fe line profile may disappear if the very innermost disc material becomes completely ionised (see Nandra et al. 1997b), consistent with our observation of Mrk 205, which we note is $20 \times$ more luminous than the Seyfert 1 MCG -6-30-15.

It remains conceivable that the "broad" line does not originate from an accretion disc. A spherical rather than planar geometry could produce the observed profile, with the blue peak being produced naturally through transverse Doppler motion. Indeed the first formulation of X-ray reflection (Guilbert \& Rees 1988) assumed a spherical distribution of clouds. Again we could be observing a blend of several Fe lines, from different ionised species and recall this model gave an equally good fit to the data (see fit 7). One possibility is the ionised lines originate from the warm electron scattering region associated with Seyfert 2 galaxies, which can produce substantial emission from $\mathrm{He}$ and $\mathrm{H}$-like iron (Krolik \& Kallman 1987). We note the spectrum of the Compton-thick Seyfert 2, NGC 1068, shows strong line emission from neutral, He-like and $\mathrm{H}-$ like iron (Marshall et al. 1993; Iwasawa et al. 1997). The difference in Mrk 205, where we observe the direct nuclear $\mathrm{X}$-ray continuum, is that the equivalent widths of the lines are considerably smaller. 


\section{Conclusions}

Observations with $X M M-N$ ewton have revealed a remarkable iron $\mathrm{K}$ line profile in the low luminosity quasar Mrk 205. The broad iron line component at $6.7 \mathrm{keV}$ is inconsistent with the relativistic profile expected from the inner accretion disc (Fabian et al. 1989), unless the disc material is highly ionised. The narrow line at $6.4 \mathrm{keV}$ probably arises from Compton scattering off distant cool material; we may infact be observing the reflected X-ray emission from the putative molecular torus in Mrk 205.

Acknowledgements. This work is based on observations obtained with XMM-Newton, an ESA science mission with instruments and contributions directly funded by ESA Member States and the USA (NASA). EPIC was developed by the EPIC Consortium led by the Principal Investigator, Dr. M. J. L. Turner. The consortium comprises the following Institutes: University of Leicester, University of Birmingham (UK); CEA/Saclay, IAS Orsay, CESR Toulouse (France); IAAP Tuebingen, MPE Garching (Germany); IFC Milan, ITESRE Bologna, OAPA Palermo, Italy. EPIC is funded by: PPARC, CEA, CNES, DLR and ASI. The authors would also like to thank the EPIC instrument team, for their hard work during the calibration phase and the SOC and SSC teams for making the observation and subsequent analysis possible. It is a pleasure to thank Gareth Griffiths, Steve Sembay and Richard West for their help and advice since the launch. We also thank the referee (Paul Nandra) for providing a rapid reply to this paper and for some useful comments and suggestions.

\section{References}

Antonucci, R. 1993, ARA\&A, 31, 473

Bahcall, J. N., Jannuzi, B. T., Schneider, D. P., Hartig, G. F. \& Jenkins, E. B. 1992, ApJ, 398, 495

Blackman, E. G. 1999, MNRAS, 306, L25

Bowen, D. V., \& Blades, J. C. 1993, ApJ, 403, L55

Elvis, M., Lockman, F. J., \& Wilkes, B. J. 1989, ApJ, 97, 777

Fabian, A. C., Rees, M. J., Stella, L., \& White, N. E. 1989, MNRAS, 238, 729

George, I. M., \& Fabian, A. C. 1991, MNRAS, 249, 352
Guilbert, P. W., \& Rees, M. J. 1988, MNRAS, 233, 475

Iwasawa, K., \& Taniguchi, Y. 1993, ApJ, 413, L15

Iwasawa, K., Fabian, A. C., \& Matt, G. 1997, MNRAS, 289, 443

Krolik, J. H., \& Kallman, T. R. 1987, ApJ, 320, L5

Krolik, J. H., Madau, P., \& Zycki, P. T. 1994, ApJ, 420, L57

Lawson, A. J., \& Turner, M. J. L. 1997, MNRAS, 288, 920

Leahy, D. A., \& Creighton, J. 1993, MNRAS, 263, 314

Magdziarz, P., \& Zdziarski, A. A. 1995, MNRAS, 273, 837

Malkan, M., \& Sargent, W. 1982, ApJ, 254, 22

Marshall, F. E., Netzer, H., Arnaud, K. A., et al. 1993, ApJ, 405, 168

Matt, G., Fabian, A. C., \& Ross, R. R. 1993, MNRAS, 262, 179

McDowell, J. C., Elvis, M., Wilkes, B. J., et al. 1989, ApJ, 345, L13

Nandra, K., \& Pounds, K. A. 1994, MNRAS, 268, 405

Nandra, K., George, I. M., Mushotzky, R. F., Turner, T. J., \& Yaqoob, T. 1997, ApJ, 477, 602

Nandra, K., George, I. M., Mushotzky, R. F., Turner, T. J., \& Yaqoob, T. 1997b, ApJ, 488, L91

Nandra, K., George, I. M., Mushotzky, R. F., Turner, T. J., \& Yaqoob, T. 1999, ApJ, 523, L17

Nayakshin, S., Kazanas, D., \& Kallman, T. R. 2000, ApJ, 537, 833

Pounds, K. A., Nandra, K., Stewart, G. C., George, I. M., \& Fabian, A. C. 1990, Nat, 344, 132

Reeves, J. N., Turner, M. J. L., Ohashi, T. \& Kii, T. 1997, MNRAS, 292, 468

Reeves, J. N., \& Turner, M. J. L. 2000, MNRAS, 316, 234

Ross, R. R, Fabian, A. C., \& Young, A. 1999, MNRAS, 306, 461

Rush, B., Malkan, M. A., \& Edelson, R. 1996, ApJ, 473, 130

Strüder, L., Briel, U., Dennerl, K., et al. 2001, A\&A, 365, L18

Tanaka, Y., Nandra, K., Fabian, A. C., et al. 1995, Nat, 275, 659

Turner, M. J. L., Abbey, A., Arnaud, M., et al. 2001, A\&A, 365, L27

Turner, T. J., George, I. M., Nandra, K., \& Mushotzky, R. F. 1997, ApJ, 488, 164

Williams, O. R., Turner, M. J. L., Stewart, G. C., et al. 1992, ApJ, 389, 157

Yaqoob, T., George, I. M., Nandra, K., et al. 2000, ApJ, in press [astro-ph/0008471] 